\title{
NONPLASTIC TOTAL CHISEL OF THE MIDDLE EAR TO PRESERVE HEARING.
}

$\mathrm{By}$

\author{
F. OKA \\ (From the Oto-Rhino-Laryngological Department, Facult $y$ of \\ Medicine, Gumma Universit y, Maebashi)
}

The author performed operations on 19 cases of chronic epitymbanitis etc., in which the drumbead and the ossicles were intact, indicating operative treatment for preservation of hearing.

Method of operation: After chisel of the mastoid cells and the osseous bridge, the pathological products in the tympanum were thoroughly removed taking care not to injure the ossicles, drumbead and soft tissue of the external auditory meatus. Without applying plastic procedure with the soft tissue of the meatus, retro-auricular wound was closed primarily with subcutaneous mattress suture, into which a small piece of iodoform-gauze or a rubber drainage tube was inserted and removed one or two days afterward. In the auditory canal also a piece of iodoform-gauze was inserted. With assistance of suitable after-cares, as described in Japanese text, the following results were obtained: (1) There were cases of chronic suppurative epitympanitis, 9 cases of chronic suppurative epitympanitis with cholesteatoma formation, one case of chronic suppurative otitis media and another case of chronic suppurative otitis media with cholesteatoma formation. All of them were perfectly cured. (2) The healing of the retroauricular wound was perfect cosmetically and the auditory canal remains unchanged as normal and did not give rise to crust formation. (3) Days required until perfect cure: 7 94 days at both extremes averaging 31 days; penicillin was used in 9 cases, which were cured in between 13 and 94 days, with an average of 31 days. (4) Hearing: unchanged in 9 cases, improved in 6 cases and slightly impaired in 4 cases, while no case aggravated. (5) All cases with one exception were free from relapse so far, after more than one year. 